\title{
RELACIÓN ENTRE EL NIVEL DE LUMINOSIDAD EN UNA UNIDAD DE CUIDADO INTENSIVO NEONATAL Y LA RETINOPATÍA DE LA PREMATURIDAD LIMA PERU-2018
}

\author{
Augusto Bernadino Chafloque Cervantes ${ }^{a}$, Evelyn Margot Aspajo Tejada ${ }^{\mathrm{b}}$.
}

\begin{abstract}
RESUMEN
Objetivo: Determinar la relación entre luminosidad ambiente y retinopatía de la prematuridad en recién nacidos de muy bajo peso al nacer. Metodología: el presente estudio es prospectivo, experimental, longitudinal de tipo cohorte que comprende a todos los prematuros de muy bajo peso al nacer, nacidos en el año 2018. Se formaron dos grupos de estudio, uno expuesto a la luz natural del ambiente y otro grupo no expuesto a los que se les cubrió los ojos con antifaz de tela negra. Ambos grupos fueron homogéneos según edad, sexo y peso. Los datos fueron analizados con pruebas Chi-cuadrado y Mantel-Haenzsel con nivel de confianza del $95 \%$. Resultados: El $57.6 \%$ de los pacientes expuestos y un $24.4 \%$ de los pacientes no expuestos desarrollo retinopatía de la prematuridad sin mostrar diferencia significativa en su incidencia. Sin embargo, cuando se analizó el grado o severidad de la ROP si se encontró significancia estadística. Conclusión: La luz no es un factor de riesgo para desarrollar retinopatía de la prematuridad; sin embargo, la luz ambiental si se relaciona con la presencia de una severidad mayor de la enfermedad.
\end{abstract}

PALABRAS CLAVES: Retinopatía de la prematuridad; Oclusión ocular; Luz (Fuente: DeCS BIREME).

\section{RELATIONSHIP BETWEEN THE LUMINOSITY LEVEL IN A NEONATAL INTENSIVE CARE UNIT AND RETINOPATHY OF PREMATURITY LIMA PERU-2018}

\begin{abstract}
Objective: Determine the relationship between the brightness level in a neonatal intensive care unit and retinopathy of prematurity (ROP) in very low birth weight new born babies. Materials and methods: A experimental, longitudinal cohort was performed. It comparise the whole very low birh weight preterm new born babies, born in 2018. Randomly two study groups were formed, one exposed to ambient light and the second not exposed to the light by coverng their eyes with a black mask. Both groups were homogeneus according to age, gender and weight and their fundus was examinated weekly during 5 weeks. The data was analized with Chi square test and Mantel - Haenzsel test with a level of confidence of $95 \%$. Results: $57.6 \%$ of the exposed patients and $24.4 \%$ of the no exposed patients developed retinopaty of prematurity, no significative diference was found. Unlike the severity of ROP wich showed statistical significance related to the ocular oclussion. Conclusions: the light is not a risk factor to develop ROP, however it is asociatted to a higer severity of this disease.
\end{abstract}

Key words: Retinopaty of prematurity; Ocular occlusion; Light (Source: MeSH NLM).

\section{INTRODUCCIÓN}

La Retinopatía de la Prematuridad (ROP) es vítreoretinopatía vaso-proliferativa una patología de etiología desconocida que acontece en recién nacidos prematuros ${ }^{1}$, se ha demostrado que la raza negra presenta una menor incidencia, a diferencia de la raza oriental con una mayor tendencia ${ }^{2}$.

A pesar de los adelantos que se realizan en el tratamiento, La ROP causa pérdida de visión en muchos lactantes prematuros $^{3}$ la exposición de la retina a la luz del ambiente después del nacimiento de un prematuro, ha sido una hipótesis natural, ya que el útero es un ambiente oscuro ${ }^{4}$. La incidencia es mayor mientras menor es el peso de nacimiento y la edad gestacional del recién nacido ${ }^{5}{ }^{6}$.

Se ha reportado una relación inversamente proporcional entre el peso al nacer y la incidencia de ROP, donde en nacimientos <1,500gr., la incidencia de ROP oscila entre $24 \%-50 \%$; alcanzando hasta $90 \%$ en los neonatos con pesos <750gr. ${ }^{7}$; esto aunado al incremento en la sobrevida de recién nacidos de extremo bajo peso al nacer en los últimos años, resultado en un aumento en

\footnotetext{
a Médico Oftalmólogo del Instituto Nacional Materno Perinatal Optómetra del Instituto Nacional Materno Perinatal

Citar como: Chafloque A, Aspajo E. Relación entre el nivel de luminosidad en una unidad de cuidado intensivo neonatal y la retinopatía de la prematuridad Lima peru-2018. Rev Peru Investig Matern Perinat 2019; 8(4): 9-13

DOI https://doi.org/10.33421/inmp.2019167
} 
el numero casos de ROP como efecto colateral ${ }^{4}{ }^{8}$, lo que nos lleva a la necesidad de identificar potenciales factores riesgo para la toma de medidas preventivas ${ }^{9}$.

En nuestra revisión bibliográfica se encontraron discrepancias de resultados, donde estudios recientes han encontrado asociación entre la exposición a la luz y la incidencia de ROP ${ }^{10}$, mientras que otros estudios no encuentran relación mencionando que la luminosidad se comporta como un factor independiente en el curso de ésta disfunción vascular ${ }^{11}$.

En el nuestro medio no se han realizado estudios al respecto, por lo que siendo el objetivo principal del estudio determinar la relación entre la luz ambiental y la incidencia de ROP en recién nacidos de MBPN, se busca contribuir con evidencia epidemiológica nacional para considerar la toma de medidas preventivas en el área de cuidados intensivos neonatales.

\section{MATERIALES Y MÉTODOS}

Se realizó un estudio experimental con ciego simple, prospectivo, longitudinal de tipo cohorte. Aleatoriamente se asignaron a 104 recién nacido de muy bajo peso al nacer la oclusión ocular (grupo estudio) y a 97 recién nacidos de muy bajo peso al nacer no (grupo control). Se realizó seguimiento de fondo de ojo semanal a partir de la semana 32 de edad gestacional, hasta la semana 36 , haciendo un total de 5 exámenes por cada paciente. Se identificaron los candidatos para el estudio del área de Cuidado Intensivos Neonatales A (UCIN-A) y Unidad de Cuidados Intensivos Neonatales B (UCIN- B). Se seleccionó el grupo de estudio por medio de incubadoras asignadas al azar y se procedió a la oclusión ocular de los pacientes seleccionados al azar previo consentimiento informado 24 horas después del nacimiento con un antifaz de tela negra.

El procedimiento de oclusión consistió en colocar un antifaz de tela negra, las 24 horas del día de 3 a 4 semanas o hasta llegar a la semana 32 de gestación retirándoles el antifaz únicamente durante la hora del examen de fordo e ojo. El examen de fondo se realizó en la sala de $\mathrm{UCI}$ neonatal por un único examinador quién en todos los casos no conocía la filiación de cada prematuro examinado. El diagnóstico del estado del fondo de ojo se hizo con oftalmoscopio binocular indirecto y una lupa de 20 dioptrías positivas. Previamente se dilató la pupila con gotas tropicamida al $0,5 \%$ y fenilefrina al $2,5 \%$ mezcladas en partes iguales (1:1) siendo la dosis de 1 gota cada 15 minutos en cada ojo por 3 veces. Ambos grupo mantuvieron en todo momento condiciones similares (luz, temperatura, humedad, personal, enfermería especializada, tiempo de luz - oscuridad, alimentación, cambio de pañales) diferenciándose solo en la oclusión ocular. Los datos obtenidos se fueron registrando en una hoja de registro de historias clínicas adjunto al consentimiento informado, una ficha de cribaje semanal oftalmológico y una hoja CLAP (Consejo Latino Americano Perinatología). Cada evaluación oftalmológica fue acompañada por la medida de la luz ambiental mediante un Luxómetro la cual se realizaba antes del examen por la mañana y después del examen por la tarde.

Para el análisis estadístico se utilizó los test Chi-cuadrado y Mantel-Haenszel (significancia estadística con un 95\% de confianza), con la ayuda del programa estadístico fue el SPSS versión 20.

\section{RESULTADOS}

Durante la investigación la luz ambiental y luminaria fue de $1,200 \pm 100$ lux en promedio tanto en primer semestre como en segundo semestre del año.

El análisis bivariado, nos muestra que no existe relación entre sexo y exposición a la luz, puesto que $(p=0.295)$, nos indica que se tiene porcentajes similares de ocurrencia en el sexo masculino entre aquellos recién nacidos ocluidos (54.8\%) y no ocluidos $(47.4 \%)$, de igual manera con el sexo femenino. Igualmente para el peso del recién nacido y exposición a la luz $(p=0.642)$, es decir, se tiene porcentajes similares de ocurrencia en el grupo de peso [600-749] entre aquellos recién nacidos ocluidos (8.7\%) y no ocluidos (7.2\%); peso [750-999] entre aquellos recién nacidos ocluidos (31.7\%) y no ocluidos (39.2\%); peso [1000-1249] entre aquellos recién nacidos ocluidos $(41.3 \%)$ y no ocluidos $(34.0 \%)$ y peso [1250-1300] entre aquellos recién nacidos ocluidos (18.3\%) y no ocluidos (19.6\%). Sin embargo, existe cierta relación entre edad gestacional y exposición a la luz $(p=0.045)$. (Tabla 1$)$

El análisis inferencial determino que con un nivel de confianza del $95 \%$ se puede concluir que la exposición a la luz ambiental no se relaciona con la presencia de algún grado de ROP I o II $(p=0.282)$, es decir, el porcentaje de ROP I o II que fueron expuestos a la luz (57.6\%) es similar a los que tuvieron diagnóstico de fondo de ojo normal $(49.3 \%)$. (Tabla 2 )

Encontramos que la mayor frecuencia relativa de exposición a la luz la tienen los de ROP severo $72 \%$, comparado con ROP leve $47.7 \%$ y fondo de ojo normal $49.3 \%$. Esto nos plantea averiguar la relación con los casos que llegaron a ser de retinopatía severa (grado II). Comparando los casos de retinopatía severa con aquellos de fondo de ojo normal, así podemos concluir con un nivel de confianza del $95 \%$ que la exposición a la luz ambiental se relaciona con la presencia grado de ROP II ( $p=0.036)$, es decir, los que fueron expuestos a la luz es mayor el porcentaje de retinopatía severa $(72.0 \%)$ a los que llegaron a tener diagnóstico de fondo de ojo normal $(49.3 \%)$.

En la Tabla $\mathrm{N}^{0} 1$ la edad gestacional se relacionaba con la variable exposición a la luz, lo cual podría influenciar como variable interviniente en el análisis bivariado entre exposición a la luz y presencia de ROP grado 2 , para ello analizamos con la prueba de Mantel-Haenszel $(p=0.091)$ para verificar la influencia de la edad gestacional con la que se comprueba que para cualquier grupo de edad gestacional no es significativo $(E G=20-26, p=0.067$; $E G=27-29, p=0.908 ; E G=30-31, p=0.059$ ). 
Tabla 1. Exposición a la luz ambiental según sexo, peso del recién nacido y edad gestacional

\begin{tabular}{|c|c|c|c|c|c|}
\hline & \multicolumn{5}{|c|}{ EXPOSICIÓN A LA LUZ AMBIENTAL } \\
\hline & \multicolumn{2}{|c|}{ si } & \multicolumn{2}{|c|}{ No } & \multirow[t]{2}{*}{ p } \\
\hline & $n$ & $\%$ & $n$ & $\%$ & \\
\hline \multicolumn{6}{|l|}{ sexo } \\
\hline Masculino & 57 & 54.8 & 46 & 47.4 & 0.295 \\
\hline Femenino & 47 & 45.2 & 51 & 52.6 & \\
\hline \multicolumn{6}{|l|}{ Peso del RN } \\
\hline $600-749$ & 9 & 8.7 & 7 & 7.2 & 0.642 \\
\hline $750-999$ & 33 & 31.7 & 38 & 39.2 & \\
\hline $1000-1249$ & 43 & 41.3 & 33 & 34 & \\
\hline $1250-1500$ & 19 & 18.3 & 19 & 19.6 & \\
\hline \multicolumn{6}{|l|}{ Edad gestacional } \\
\hline $24-26$ & 5 & 4.8 & 12 & 12.4 & 0.046 \\
\hline $27-29$ & 41 & 39.4 & 45 & 46.4 & \\
\hline $30-31$ & 58 & 55.8 & 40 & 41.2 & \\
\hline
\end{tabular}

Fuente: propia

$\left.{ }^{*}\right)$ Test de X2

Tabla 2. Relación entre exposición a la luz ambiental y diagnóstico de fondo de ojo

\begin{tabular}{|c|c|c|c|c|c|}
\hline \multirow{3}{*}{$\begin{array}{l}\text { Exposición a la } \\
\text { luz ambiental }\end{array}$} & \multicolumn{4}{|c|}{ Diagnóstico de fondo de ojo } & \multirow{3}{*}{ Valor $p$} \\
\hline & \multicolumn{2}{|c|}{ ROP I } & \multicolumn{2}{|c|}{ ROP II } & \\
\hline & $\mathbf{n}$ & $\%$ & $\mathbf{n}$ & $\%$ & \\
\hline Si & 34 & 57.6 & 70 & 49.3 & \multirow{3}{*}{0.282} \\
\hline No & 25 & 42.4 & 72 & 50.7 & \\
\hline Total & 59 & 100 & 142 & 100 & \\
\hline
\end{tabular}

Fuente: propia

Tabla 3. Exposición a la luz ambiental y grados de ROP

\begin{tabular}{|c|c|c|c|c|c|c|c|}
\hline \multirow{3}{*}{$\begin{array}{c}\text { Exposición a la luz } \\
\text { ambiental }\end{array}$} & \multicolumn{6}{|c|}{ Grados de ROP } & \multirow{3}{*}{ Valor $p$} \\
\hline & \multicolumn{2}{|c|}{ ROP 0} & \multicolumn{2}{|c|}{ ROP I } & \multicolumn{2}{|c|}{ ROP II } & \\
\hline & $\mathbf{N}$ & $\%$ & $\mathbf{n}$ & $\%$ & $\mathbf{n}$ & $\%$ & \\
\hline $\mathrm{Si}$ & 70 & 49.3 & 16 & 47.1 & 18 & 72 & \\
\hline No & 72 & 50.7 & 18 & 52.9 & 7 & 28 & 0.093 \\
\hline Total & 142 & 100 & 34 & 100 & 25 & 100 & \\
\hline
\end{tabular}

Fuente: propia

Tabla 4. Exposición a la luz ambiental y retinopatia grado II

\begin{tabular}{|c|c|c|c|c|c|c|c|}
\hline \multirow{3}{*}{$\begin{array}{c}\text { Exposición a la luz } \\
\text { ambiental }\end{array}$} & \multicolumn{6}{|c|}{ Grados de ROP } & \multirow{3}{*}{ Valor $p$} \\
\hline & \multicolumn{2}{|c|}{ ROP 0} & \multicolumn{2}{|c|}{ ROP I } & \multicolumn{2}{|c|}{ ROP II } & \\
\hline & $\mathbf{N}$ & $\%$ & $\mathrm{n}$ & $\%$ & $\mathbf{n}$ & $\%$ & \\
\hline $\mathrm{Si}$ & 70 & 49.3 & 16 & 47.1 & 18 & 72 & \\
\hline No & 72 & 50.7 & 18 & 52.9 & 7 & 28 & 0.093 \\
\hline Total & 142 & 100 & 34 & 100 & 25 & 100 & \\
\hline
\end{tabular}

Fuente: propia 


\section{DISCUSIÓN}

Con toda la revisión realizada, escasa por cierto, se reportan resultados que niega esta posibilidad de relación. El seguimiento semanal realizados en todos los recién nacidos de muy bajo peso, en la UCIN de un Instituto Especializado de Lima ha permitido obtener una base de datos confiable en la que se ha tomado especial cuidado en la obtención de los datos en particular con el control del nivel de iluminación de la UCIN.

Los resultados obtenidos son similares a otros trabajos quienes consideran que el nivel de luz de las UCIN no es factor de riesgo para la aparición de retinopatía en prematuros de extremo bajo peso al nacer, a pesar que la luminosidad era inferior a la del presente ensayo ya que nuestro medio está situado en la línea ecuatorial. ${ }^{10} 11$

La edad gestacional al igual que en muchos trabajos es factores de riesgo para la presencia de ROP en el presente estudio, confirmándose que esta sigue siendo la más importante en la aparición de la retinopatía ${ }^{4} 124$ 1314 15. Numerosos factores de riesgo para el desarrollo de la disfunción vascular en prematuros de muy bajo peso al nacer han sido identificados en la literatura. Sin embargo, el papel del nivel de luz en el desarrollo de ésta enfermedad no ha sido abordado adecuadamente y el presente estudio polemiza con resultados que consideran que el nivel de luz en el recién nacido de muy bajo peso debe ser o no tomado en cuenta, para la presencia de ROP .En el presente estudio encontramos que no existe relación con un aumento con la incidencia de la ROP y coincide con el estudio de Reynolds quien realizó una investigación en la que se asignaron al azar lactantes de menos de 31 semanas de edad gestacional y de menos de 1251 gramos de peso al nacer ,pero al igual que en el presente estudio, no se encontraron diferencias significativas entre ambos grupos. El examen de las características de los valores basales reveló sólo diferencias mínimas entre grupos y éstas se asociaron a un riesgo ligeramente superior al esperado de ROP en el grupo control, que por lo tanto no afecta las conclusiones. ${ }^{16}$

La ROP severa se ha asociado también a otras enfermedades en las que puede estar implicada la lesión por estrés oxidativo ${ }^{6}$. Para el presente estudio el grado 2 , que es grado más severo resultó estar asociada a esta retinopatía, hallazgo que no ha sido tomado en cuenta en otros estudios.

La retina como tejido nervioso consume abundante oxígeno y su crecimiento por lo tanto es rápido, esto aunado a el exceso de luz en los prematuros y la elevada velocidad de la curva de crecimiento presente en ellos podríamos explicar de alguna manera daño en la retina y la aparición grado 2 de ROP ${ }^{17}$. Se debe realizar más investigaciones que contribuyan a disminuir la frecuencia de ésta disfunción considerada como epidemia a nivel mundial buscando prevenir sus efectos en forma precoz valorados en trabajos recientes y en donde se viene utilizando drogas que reducen la severidad ${ }^{18}$.

En conclusión: La Luxometría de la UCIN del Instituto Especializado es de 1,200 \pm 100 lux durante la mañana y la tarde. La incidencia de Retinopatía en prematuros de muy bajo peso no aumenta con la exposición a la exposición a la luz a mayor de 1,200 lux. En recién nacidos prematuros de muy bajo peso, el nivel de iluminación se relaciona con los grados de severidad de la enfermedad - ROP. El análisis el peso, la edad gestacional y el sexo no son covariables que influyan en la variable "exposición a la luz" para la aparición de ROP

Financiamiento: Concurso de investigación del INMP 2019

Conflicto de interés: Los autores declaran no tener algún conflicto de intereses

\section{REFERENCIAS BIBLIOGRÁFICAS}

1. Jefferies AL. Retinopathy of prematurity: An update on screening and management. Paediatr Child Health. marzo de 2016;21(2):101-4. PMID: 27095887 PMCID: PMC4807789

2. Laws D, Shaw DE, Robinson J, Jones HS, Ng YK, Fielder AR. Retinopathy of prematurity: a prospective study. Review at six months. Eye Lond Engl. 1992;6 ( Pt 5):477-83. DOI: 10.1038/eye.1992.101

3. Fierson WM, Ophthalmology AAOPS on, Ophthalmology AAO, Strabismus AA for POA, Orthoptists AA of C. Screening Examination of Premature Infants for Retinopathy of Prematurity. Pediatrics [Internet]. 1 de diciembre de 2018 [citado 10 de diciembre de 2019];142(6). Disponible en: https://pediatrics.aappublications.org/content/142/6/ e20183061 DOI: 10.1542/peds.2018-3061

4. The STOP-ROP Multicenter group. Supplemental Therapeutic Oxygen for Prethreshold Retinopathy Of Prematurity (STOP-ROP), a randomized, controlled trial. I: primary outcomes. Pediatrics. febrero de 2000;105(2):295310. DOI: $10.1542 /$ peds.105.2.295

5. Dani C, Reali MF, Bertini G, Martelli E, Pezzati M, Rubaltelli FF. The role of blood transfusions and iron intake on retinopathy of prematurity. Early Hum Dev. abril de 2001;62(1):57-63. DOI: 10.1016/s0378-3782(01)00115-3

6. Painter SL, Wilkinson AR, Desai P, Goldacre MJ, Patel CK. Incidence and treatment of retinopathy of prematurity in England between 1990 and 2011: database study. $\mathrm{Br} \mathrm{J}$ Ophthalmol. junio de 2015;99(6):807-11. DOI: 10.1136/ bjophthalmol-2014-305561

7. Lee SK, Normand C, McMillan D, Ohlsson A, Vincer M, Lyons $\mathrm{C}$, et al. Evidence for changing guidelines for routine screening for retinopathy of prematurity. Arch Pediatr Adolesc Med. marzo de 2001;155(3):387-95. DOI: 10.1001/ archpedi.155.3.387

8. Reynolds JD. The management of retinopathy of prematurity. Paediatr Drugs. 2001;3(4):263-72. DOI: 10.2165/00128072200103040-00003

9. Christiansen SP, Fray KJ, Spencer T. Ocular outcomes in low birth weight premature infants with intraventricular hemorrhage. J Pediatr Ophthalmol Strabismus. junio de 2002;39(3):157-65. PMID: 12051281

10. Ackerman B, Sherwonit E, Williams J. Reduced incidental light exposure: effect on the development of retinopathy of prematurity in low birth weight infants. Pediatrics. junio de 1989;83(6):958-62. PMID: 2726352

11. Watts JL. Retinopathy of prematurity: Effective care of the newborn infant. Oxford: Oxford University Press: Sinclair JC, Bracken MB; 1992. 617-639 p.

12. Isaac M, Tehrani N, Mireskandari K, Medscape. Involution patterns of retinopathy of prematurity after treatment with intravitreal bevacizumab: implications for follow-up. Eye Lond Engl. marzo de 2016;30(3):333-41. DOI: 10.1038/ eye.2015.289 
13. Deulofeut R, Critz A, Adams-Chapman I, Sola A. Avoiding hyperoxia in infants $<$ or $=1250 \mathrm{~g}$ is associated with improved short- and long-term outcomes. J Perinatol Off J Calif Perinat Assoc. noviembre de 2006;26(11):700-5. DOI: 10.1038/ sj.jp. 7211608

14. Rodríguez-Hurtado FJ, Cañizares JM. Despistaje de la retinopatía del prematuro: Nuestra experiencia sobre los límites de peso al nacer, edad gestacional y otros factores de riesgo. Arch Soc Esp Oftalmol. mayo de 2006;81(5):275-80. ISSN: 0365-6691

15. Grunauer N, Iriondo Sanz M, Serra Castanera A, Krauel Vidal J, Jiménez González R. Retinopatía del prematuro. Casuística de los años 1995-2001. An Pediatría. 1 de mayo de 2003;58(5):471-7. DOI: 10.1016/S1695-4033(03)78095-3

16. Reynolds JD, Dobson V, Quinn GE, Fielder AR, Palmer EA, Saunders RA, et al. Evidence-based screening criteria for retinopathy of prematurity: natural history data from the CRYO-ROP and LIGHT-ROP studies. Arch Ophthalmol Chic III 1960. noviembre de 2002;120(11):1470-6 DOI: 10.1001/ archopht.120.11.1470.
17. Chow LC, Wright KW, Sola A, CSMC Oxygen Administration Study Group. Can changes in clinical practice decrease the incidence of severe retinopathy of prematurity in very low birth weight infants? Pediatrics. febrero de 2003;111(2):33945. DOI: 10.1542/peds.111.2.339

18. Mintz-Hittner HA, Geloneck MM, Chuang AZ. Clinical Management of Recurrent Retinopathy of Prematurity after Intravitreal Bevacizumab Monotherapy. Ophthalmology. 2016;123(9):1845-55. DOI: 10.1016/j.ophtha.2016.04.028

\section{Correspondencia:}

Augusto Bernadino Chafloque Cervantes

Dirección: Calle Manuel del Pino \# 890 - Cercado de Lima correo: chaflocerv@hotmail.com

Teléfono: 999413584 